\title{
Noninvasive skin imaging
}

\author{
Symon D'Oyly Cotton \\ Ela Claridge
}

School of Computer Science, The University of Birmingham

Birmingham B15 2TT, UK

Per Hall

Department of Plastic Surgery, Addenbrooke's Hospital, Cambridge, UK.

Information Processing in Medical Imaging,

Duncan J and Gindi G Eds.

Lecture Notes in Computer Science 1230: Springer, 501 - 506. 


\title{
Noninvasive Skin Imaging
}

\author{
Symon Cotton ${ }^{1}$, Ela Claridge ${ }^{1}$, Per Hall ${ }^{2}$ \\ 1 School of Computer Science, University Of Birmingham, B15 2TT, UK \\ 2 Consultant plastic surgeon, Addenbrooke's Hospital, Cambridge, UK
}

\begin{abstract}
An earlier model of colour formation within normal human skin was extended to include architectural distortions associated with various pigmented skin lesions, including malignant melanoma. The extended five-layer model makes it possible to derive parameters characterising the thickness and pigment composition of the skin layers from calibrated colour and infrared images of skin lesions. The extracted parameters can be used to reconstruct a full 3-dimensional model of the skin architecture which conveys information grossly comparable to that available through microscopical examination of biopsied skin tissue.
\end{abstract}

This work forms a part of research at the University of Birmingham into developing theories and techniques which aim to aid clinicians in the early diagnosis of malignant melanoma.

Our previous paper [2] presented a model of colour formation within normal human skin. The model is based on the Kubelka-Munk theory [5] of scattering and absorption within inhomogeneous materials and the physics pertaining to their colour properties. By considering the skin to be a layered construction of such materials, the stratum corneum, epidermis, papillary dermis and reticular dermis, and by exploiting the physics related to the optical interface between these layers, the model generates all possible colours occurring within normal human skin. In particular, the model predicts that all normal skin colours lie on a simple curved surface patch within a three-dimensional colour space bounded by two physiologically meaningful axes, one corresponding to the amount of melanin within the epidermis and the other to the amount of blood within the dermis.

The model postulated that abnormal skin conditions would cause the skin colours to deviate from the predicted surface in the colour space. This paper exploits this idea further. In particular, it demonstrates that it is possible to derive detailed information about the internal skin architecture and composition grossly comparable to information available through the microscopical examination of tumour tissue but without incurring the problems inherent in obtaining a biopsy. The central premise explored here is, therefore, that as abnormal skin often has a different internal architecture to normal skin it is a fair proposition that the coloration may not be bounded to this surface; if this is true, then the nature of the deviation may yield important information about the skin architecture.

To explore this the model presented in the previous paper was extended to predict the skin coloration associated with conditions where melanocytes pen- 
etrate into the dermis ${ }^{3}$. These are commonly seen in such conditions as the benign blue nevus and invasive skin cancer, such as melanomas, often leading to the blue hues characteristic with these conditions [1]. The "form" and depth of this invasion is an extremely important diagnostic factor in determining the nature of a skin lesion and, if abnormal, the relevant treatment and prognosis; currently the only reliable method of obtaining this information is by biopsy.

To account for the architectural distortion where dermal melanocytes occupy a region of the papillary dermis the model of normal skin needed to be extended to include additional layers. As can be seen from Figure 1, there are now five distinct layers which can be combined to construct an extended model: a layer within the upper papillary dermis containing no melanin; a layer within the upper papillary dermis containing melanin; a layer within the lower papillary dermis containing melanin; and a layer within the lower papillary dermis containing no melanin and finally the epidermis. The extended model [3] specifies how the magnitude of any colour primary depends on these model parameters thus allowing exploration of the coloration expected for various skin conditions.

A first such "computational experiment" modelled in vitro dermal tissue, that is bloodless skin with the epidermis removed. This analysis showed that the coloration "does indeed move off the surface corresponding to normal skin" in situations where melanocytes have penetrated the dermis. Further analysis led to the conclusion that "In principle, therefore, if presented with a section of in vitro dermis it should be possible to assess both the presence, concentration and position of melanocytes by an examination of the coloration" [3].

For such an approach to be useful when applied to living, in vivo, tissue it was necessary to include the effect of both melanin absorption within the epidermis and blood within the dermis. The result of this analysis showed that although the amount of blood could be quantified directly through an analysis of the remitted skin colour, when combined with dermal penetration of melanocytes, the amount of epidermal melanin could not. It was, however, possible to ascertain that "an amount" of dermal invasion had occurred, thus allowing areas of normal and abnormal skin to be segmented, but it was impossible to unravel the relative amounts of each.

To gain insight into the internal architecture therefore requires that either the amount of epidermal melanin, penetration of dermal melanocytes or the concentration of these melanocytes are known. If one of these parameters can be specified then it becomes possible to disentangle the other two from a measure of the coloration. Through further research it is hoped to be able to measure one of these parameters directly for every point within a lesion image. At present, however, the following approach has been adopted. First, the normal areas of skin are identified - that is areas with no dermal penetration of melanocytes and within these areas the amount of epidermal melanin is ascertained; this is then followed by an interpolation of these surrounding melanin values into the abnormal areas. This approach assumes that the amount of epidermal melanin does not change by a significant amount within these areas or if it does it varies

\footnotetext{
${ }^{3}$ In normal skin melanocytes are restricted to the epidermis.
} 
in a predictable manner thus allowing the variation to be modelled.

As described in [3] however it is necessary to ascertain one further parameter before such analysis can be undertaken. This parameter, the papillary dermal thickness, has a pronounced effect on the light remitted from a skin lesion. Indeed the change in coloration due to a variation in this parameter is almost identical to that due to melanocytic descent thus leading to lesions only varying in this parameter being wrongly classified. As an example it is possible to find malignant invasive melanomas with an identical coloration to that of simple warts.

This result casts doubt on the effectiveness of using purely colour information in the diagnosis of malignant melanoma and may offer itself as an explanation for the "moderate success achieved" by Umbaugh et al. [6] when they attempted to classify lesion types by an investigation of coloration. However, this is not to dismiss the usefulness of colour information when combined with other extracted lesion features such as that demonstrated by Dhawan and Sicsu [4] when they combined colour with a texture analysis; and Umbaugh et al. [7] when they applied artificial intelligence techniques to variations in lesion colour.

From the extended skin model the variation of remitted light with papillary dermal thickness can be ascertained. Therefore if this thickness were known for each image point it should be possible to calculate a transformation that adjusts the measured coloration to that of any specified papillary dermal thickness thus removing the metameric problems previously discussed.

The problem, therefore, is how can the papillary dermal thickness be measured noninvasively? In formulating a solution to this problem it is useful to recall that the amount of light remitted from the skin becomes highly dependent upon this factor as the wavelength increases [3]. When this is combined with the observation that both melanin and blood absorption drop significantly with increasing wavelength it seems it may be possible to find a wavelength range where the amount of remitted light depends largely on the papillary dermal thickness. This is never the case within the visible portion of the spectrum. However, it becomes plausible if the considered wavelength range is extended into the infrared. For instance, in the wavelength range 600-800 $\mathrm{nm}$ the absorption of melanin drops to around one tenth of its peak within the visible portion of the spectrum; the absorption of blood drops by around a factor of a hundred whilst the sensitivity of remitted light to variations in papillary dermal thickness increases. This difference becomes even more marked as longer wavelengths are considered; for example, the absorption of melanin drops by a further order of magnitude in the wavelength range $800-1000 \mathrm{~nm}$. Indeed, within these wavelength ranges the thickness of the papillary dermis is the major parameter affecting skin coloration.

As these wavelength ranges are easily accessible with existing infrared film and infrared digital cameras it should be possible to use this information to provide the desired calibration. As an example of how this may be performed consider Figure 1 where the intensity of remitted light for in vivo skin between $600-800 \mathrm{~nm}$ is plotted against that measured between $800-1000 \mathrm{~nm}$. As can be seen from this graph the amount of remitted light falls, with increasing melanin, faster for the $600-800 \mathrm{~nm}$ primary than for the $800-1000 \mathrm{~nm}$ primary. This is 
as one would expect; more interesting, however, is the significant variation in both primaries with papillary dermal thickness. This observation then allows construction of the lines of constant papillary dermal thickness as shown in the graph. A measurement of this thickness parameter can thus be recovered by obtaining images acquired within the given wavelength ranges and looking-up the corresponding papillary dermal thickness from the graph.
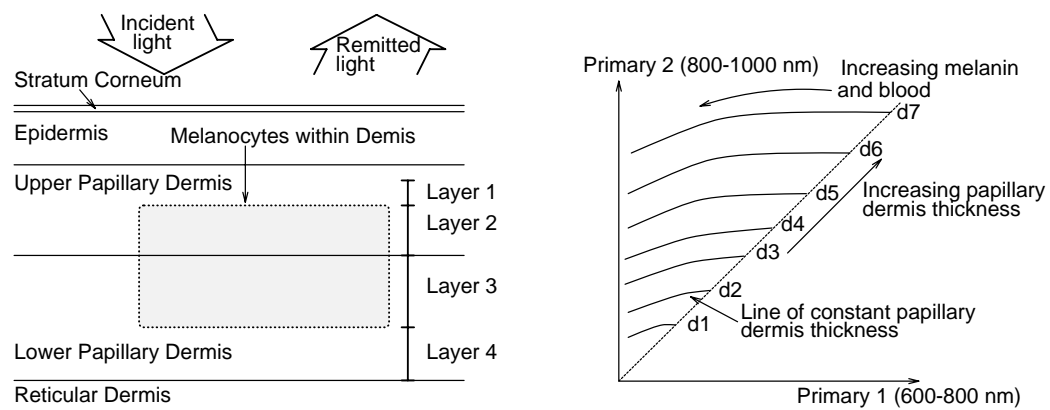

Fig. 1. Left: Extended skin model; Right: Variation of infrared primary intensities with epidermal melanin, blood, and papillary dermal thickness

The ideas outlined above give theoretical foundations for development of a noninvasive system capable of extracting information about the internal architecture of human skin. The complete process can be summarised as follows. Firstly, skin images are obtained using two infrared primaries and from these the thickness of the papillary dermal layer is computed across the image. This measurement is then used to "calibrate" the image so that the coloration for each image point is adjusted to that of a standard papillary dermal thickness ${ }^{4}$. Abnormal regions within the image can now be identified as they deviate, in a three dimensional colour space, from the surface of expected coloration for skin at this standard papillary dermal thickness. For those points identified as normal the amount of epidermal melanin can now be computed from the position, of their coloration, on this surface. By a process of interpolation, and possibly the use of a model, the amount of epidermal melanin within the abnormal regions is estimated. The points identified as abnormal can now be compared with those generated by the theoretical model for different concentrations and positions of melanocytes within the dermis as well as for differing amounts of dermal blood and these parameters quantified.

At the end of this process we now have information pertaining to the amount of epidermal melanin, dermal blood, thickness of the papillary dermis along with

\footnotetext{
${ }^{4}$ Even at this early stage, with the perturbing effect of variations in papillary dermal thickness removed, the calibrated images should prove useful to a clinician.
} 
the concentration and position of dermal melanocytes for each point within the image. This can be presented using a number of methods ranging from simply reporting the maximum depth to which melanocytes have descended within the dermis to using the data to generate either a cross section, or a fully three dimensional representation, of the internal skin structure.

As a pre-cursor to a clinical trial the effectiveness of the system was assessed using computer generated phantoms of various skin lesions. Given knowledge of the internal structure of various skin conditions the appearance of the lesion was generated by the extended model producing both colour RGB images and infrared images. As examples of this consider the cross sectional representations, (b) and (f), of the two skin lesions shown in Figure 2. Within the left hand lesion, lesion $\mathrm{A}$, there is no dermal invasion of melanocytes with the only architectural point of note being the thinned papillary dermis. The right hand lesion, lesion B, however represents a blue nevus with melanocytes, the dark area in the center of cross section (f), existing within the lower section of the papillary dermis. Although these lesions have markedly different internal architectures the observed coloration predicted by the extended model within the RGB colour space ${ }^{5}$, (a) and (e), are very similar. It is this similarity which leads to problems when a diagnostic technique uses this coloration as its sole input.

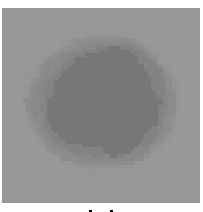

(a)

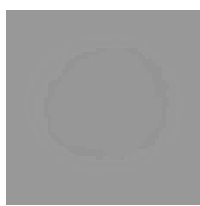

(c)

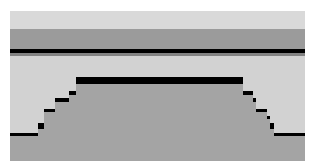

(b)

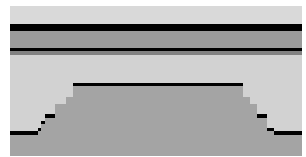

(d)

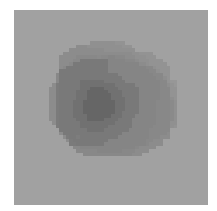

(e)

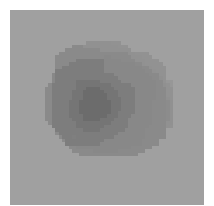

(g)

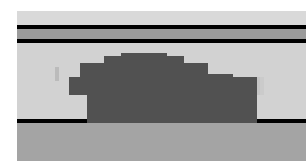

(f)

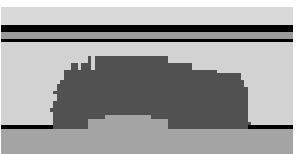

(h)

Fig. 2. Left: (a) RGB image of lesion A calculated from (b) internal structure input to system; (c) RGB image of lesion A calibrated to constant papillary thickness and (d) internal structure calculated by system; Right: (e) ... (h) the same for lesion B

After the infrared images are obtained the papillary dermis thickness can be ascertained for all points within the image. This then allows calibration of the lesion images to that of a standard papillary dermal thickness resulting in images (c) and (g) seen in Figure 2. As can be seen the appearance of lesion A has changed markedly which is as would be expected given that the major architectural feature within this lesion was a thinned papillary dermis. Given

\footnotetext{
${ }^{5}$ These figures are black and white representations of the colour images.
} 
that the images are now calibrated to a standard papillary dermal thickness, processing can now proceed as described above resulting in measures for the parameters outlined earlier. These are shown within Figure 2 as cross sections (d) and (h) comparing favourably with the actual internal structure (b) and (f).

The work presented here outlines a noninvasive system allowing the investigation of internal skin architecture. It has been shown that provided with one standard colour and two infrared images, the developed system can recover a number of parameters characterising skin structure, including the amount of epidermal melanin, dermal blood, papillary dermal thickness along with the position and concentration of melanocytes within the dermis.

This work is of both theoretical and practical importance. The theoretical model of interaction of light with a multi-layer optical system, which is at the foundation of this work, can serve as a powerful simulation system for other medical applications. Moreover, the model can generate explanations and thus increase understanding of why skin diseases manifest themselves through particular appearances. Examples include malignant melanoma, port wine stains, naevi, wound healing etc. The practical importance of this work has two strands. Firstly, it gives clinical researchers a powerful and noninvasive tool with which to study skin diseases. At present dermatologists have a very limited choice of noninvasive tools with which to examine skin conditions and methods, such as biopsy, which are invasive and can sometimes adversely affect the outcome of the disease (as, for example, in malignant melanoma). Consequently, knowledge about the physical progress of the disease can be scant. Secondly, the system can be used as an aid in the diagnosis of skin diseases such as melanoma; the importance of this cannot be overstressed. A clinical trial is currently being undertaken at Addenbrooke's hospital in Cambridge where the predicted internal architecture generated by the system will be compared with the actual architecture found through biopsy.

\section{References}

1. Anderson, R., Parrish, B. S., Parrish, J. A.: The Optics of Human Skin. The Journal of Investigative Dermatology 77(1) (1981) 13-19

2. Cotton, S. D., Claridge, E.: Developing a Predictive Model of Human Skin Colouring. SPIE Proceedings Vol. 2708 Medical Imaging 1996: Physics of Medical Imaging (April 1996) 814-825

3. Cotton, S. D., Claridge, E.: A Noninvasive Skin Imaging System. Technical Report CSR-97-3. University of Birmingham School of Computer Science (1997)

4. Dhawan, A. P., Sicsu, A.: Segmentation of Images of Skin Lesions Using Color and Texture Information of Surface Pigmentation. Computerized Medical Imaging and Graphics 16(3) (1992) 163-177

5. Egan, W. G., Hilgeman, T. W.: Optical Properties of Inhomogeneous Materials. Academic Press (1979)

6. Umbaugh, S. E., Moss, R. H., Stoecker, W. V.: Automatic Color Segmentation of Images with Application to Detection of Variegated Coloring in Skin Tumors. IEEE Engineering in Medicine and Biology Magazine (December 1989) 43-52 
7. Umbaugh, S. E., Moss, R. H., Stoecker, W. V.: Applying Artificial Intelligence to the Identification of Variegated Coloring in Skin Tumors. IEEE Engineering in Medicine and Biology Magazine (December 1991) 57-62

This article was processed using the $\mathrm{IAT}_{\mathrm{E}} \mathrm{X}$ macro package with LLNCS style 